\title{
The moisture movement of natural aggregate and its effect on concrete*
}

\author{
by L. C. Snowdon, B.Sc. and A. G. Edwards, B.Sc.
}

\section{Contribution by J. W. Dougill \\ (University of London, Imperial College of Science and Technology)}

The conclusions reached by the authors in their investigation corroborate those of other workers considering similar effects due to thermal strains. It is clear that, if deterioration due to weathering is considered to be due either to fracture of the mortar matrix or to the break-down of bond between the aggregate particles and the mortar, the likelihood of failure is determined by the magnitude of the interparticle stresses developed.

If a small representative piece of concrete situated inside a specimen subjected to shrinkage is considered, the stresses acting at the boundaries of this element will be those caused by the over-all shrinkage and calculated by conventional methods using the properties of the concrete. In addition to the interparticle stresses within the element caused by this loading, there will be the stresses caused by differential shrinkage between the aggregate and the mortar matrix. The conventionally determined stresses thus constitute the loading on the element whereas variations in strength are caused by the stresses due to differential shrinkage. In general, both the shrinkage differential and the overall shrinkage must be considered in assessing the probability of failure and it is suggested that in practice the former may be as important as the latter.

The writer has studied the variations in strength, caused by differential shrinkage, by means of a simplified structural model for concrete and it is apparent that, if the unrestrained shrinkage of the mortar is greater than that of the aggregate, both the unconfined compressive and the tensile strengths are reduced. Lea and Stradling had previously found this to be so in their study of the fire resistance of concrete.

In a test on a long thin concrete specimen, shrinkage by drying will occur most rapidly at the outer faces and will gradually spread further into the specimen. The longitudinal tensile stresses at the surface will first increase with increasing shrinkage strain and then decrease, disappearing when the unrestrained shrinkage of the concrete is the same throughout. The strength of the concrete at the surface will be decreased until it reaches a minimum corresponding to the maximum differential shrinkage. The variation of surface stress and strength with time will be as shown in

-Pages 109-116 of Magazine No. 41.

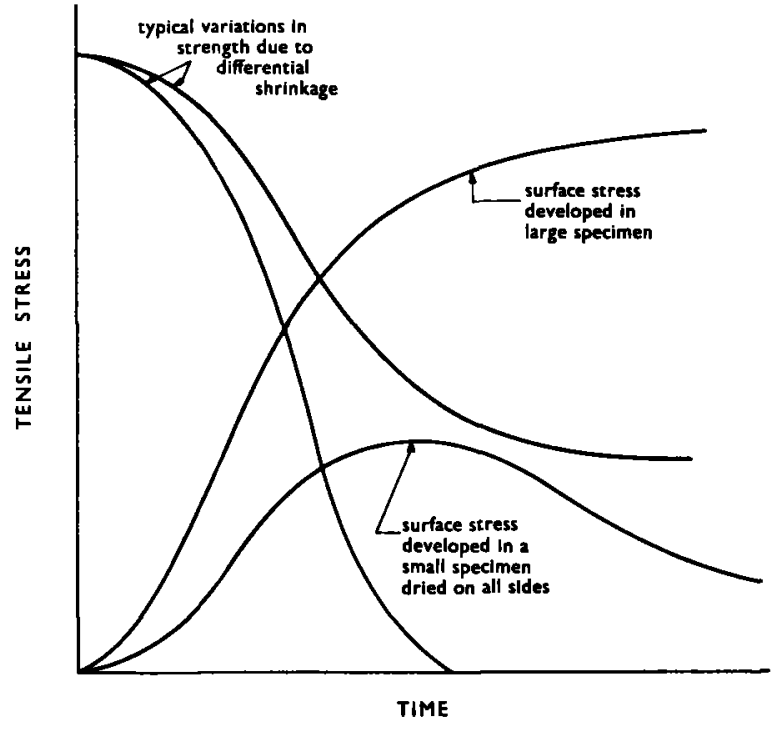

Figure I

Figure I. It appears that cracking is most likely to occur some time after exposure and that, if the specimen survives, the likelihood of failure is decreased as shrinkage diffuses further into the specimen.

The occurrence of a similar reduction in stress with time may explain the recovery, mentioned in the paper, of certain of the specimens tested. However, the actual exposure conditions must be more complex than those now considered and continued hydration may also assist the recovery.

With a very small specimen, the final state will be reached very quickly and at no time will the conventionally determined stresses be very large. In this case, the possibility of failure will be determined almost entirely by the effect of the shrinkage differential on strength. When a large piece of concrete is drying out, however, the shrinkage is almost completely restrained and the shrinkage stress at the surface will continue to increase until either failure occurs or the unrestrained shrinkage strain reaches a maximum. In this case, the possibility of failure may be determined as much by the shrinkage of the concrete as by the shrinkage differential between the constituents.

The results given in Table 4 of the paper for the shrinkage of sand-cement and sand-lime-cement mortars are particularly interesting. Owing to the expansion accompanying the hydration of the lime, the introduction of lime with additional water into a mortar mix reduces both the over-all shrinkage and the 
shrinkage differential between the sand particles and the cement paste. Shrinkage can be a problem in the use of no-fines concrete, especially when wall panels are restrained by surrounding dense concrete beams and columns. It is possible that this problem could be considerably reduced by the inclusion of a quantity of lime in no-fines concrete mixes.

\section{Reply by the authors}

We wish to thank Mr Dougill for his interesting and corroborative contribution. As Mr Dougill rightly says, the cracking of exposed concrete must depend on the magnitude of the internal stresses set up by shrinkage or thermal differentials or by both and, further, the existence of this internal state of stress must also adversely affect the compressive or tensile strengths as traditionally determined. There is of course no critical value of internal stress which will initiate cracking; cracking will occur when the stress set up exceeds the ultimate stress that the concrete has been able to develop up to that instant. In other words it is a race against time between developing destructive forces and developing strength. If the latter wins, the concrete remains sound: if it loses, the concrete fails. It is for this reason that prolonged wet-curing is advocated-a process which promotes optimum ultimate strength in the concrete before it is stressed by developing differentials.

It is noted that Mr Dougill's studies show that a reduction in intrinsic strength will always occur when the shrinkage of the matrix is greater than that of the aggregate-a prediction made much earlier by Lea and Stradling - but, surely, these conditions must nearly always obtain. However, if it were possible for the aggregate shrinkage to exceed that of the matrix we should still expect some reduction in strength to take place. Again, even with equality of ultimate shrinkage between aggregate and matrix, internal stresses will still occur if the two materials do not follow the same cycle of moisture loss and gain.

The authors agree that some recovery of cracking could be expected as a result of the decrease in differential brought about as the value of shrinkage in the core concrete approaches that of the surface concrete. They would also agree that with small specimens differential shrinkage between the constituents is the principal criterion for failure but with large specimens failure may depend as much on the shrinkage gradient between core and surface.

The interesting reduction in the shrinkage of sandcement mixes reported in Table 4 can hardly be attributed to the hydration of the lime as Mr Dougill suggests, since the added lime as well as that liberated by the setting cement is already hydrated. Later on the carbonation of the hydrate in the surface layers leads to further shrinkage. It seems likely that the change is in fact brought about by a change in the physical properties of the lime-cement matrix.

The use of added lime to combat the effects of high shrinkage in no-fines concrete is a novel one and might be worth putting to the test. 